\title{
Particle Deposition in Human Lung Airways: Effects of Airflow, Particle Size, and Mechanisms
}

\author{
Cuiyun Ou ${ }^{1,2 *}$ Jian Hang ${ }^{1,2}$, Qihong Deng ${ }^{3,4}$ \\ ${ }^{1}$ School of Atmospheric Sciences, Guangdong Province Key Laboratory for Climate Change and Natural Disaster Studies, \\ Sun Yat-sen University, Guangzhou 510275, China \\ ${ }^{2}$ Southern Marine Science and Engineering Guangdong Laboratory (Zhuhai), Zhuhai, China \\ ${ }^{3}$ School of Energy Science and Engineering, Central South University, Changsha 410083, China \\ ${ }^{4}$ XiangYa School of Public Health, Central South University, Changsha 410013, China
}

\begin{abstract}
Characterizing particle deposition in the airways of the human lungs is essential to evaluate the health effects of particulate air pollution. However, lung deposition is rather complicated, and its main influencing factors remain unclear. Hence, this study applied computational fluid dynamics (CFD) to investigate the roles of airflow (Reynolds number [Re] $=100-2000$ ) and particle size $(1-10 \mu \mathrm{m})$ in deposition using a human tracheobronchial airway model (G3-G6). We calculated the deposition efficiency (DE) based on two mechanisms, inertial impaction $\left(\mathrm{DE}_{\mathrm{i}}\right)$ and gravitational sedimentation $\left(\mathrm{DE}_{\mathrm{g}}\right)$, which produced hot spots around the bifurcations and uniform distributions along the tube, respectively. Furthermore, as the particle size increased, $\mathrm{DE}_{\mathrm{i}}$ grew rapidly, whereas $\mathrm{DE}_{\mathrm{g}}$ grew log-linearly. Particles that were less than $2 \mu \mathrm{m}$ in diameter $\mu$ only penetrated deep in the lungs where the airflow rate was low, but $3 \mu \mathrm{m}$ particles were more likely to settle in this region owing to the combination of gravitational sedimentation and inertial impaction. Larger particles, on the other hand, mainly deposited in the proximal bifurcations as a result of inertial impaction. Additionally, the deposition due to inertial impaction and that due to gravitational sedimentation primarily depended on the Stokes number $\left(S_{t}\right)$ and the ratio of $S_{t}$ to $R^{2}$, respectively. The orientation of the human body was another potential factor in the pattern of deposition, although the upright and lateral positions exhibited similar deposition efficiencies for $3 \mu \mathrm{m}$ particles regardless of Re. These findings identify the critical Reynolds number at which the particle deposition mechanism for a specific size shifts from gravitation to inertia.
\end{abstract}

Keywords: Inhalable particle; CFD simulation; Airflow pattern; Particle deposition; Human body orientation; Deposition mechanisms.

\section{INTRODUCTION}

A predictive understanding of particle transport through bifurcating airways is essential either to optimize therapeutic effects of pharmaceutical aerosols or to minimize harmful effects of toxic aerosol exposure (Patton and Byron, 2007; Finlay and Martin, 2008; Löndahl et al., 2017; Deng et al., 2018). However, particle deposition in human lung is rather complicated (Deng et al., 2019). The mechanism of specific diameter particle deposition in the designated human lung regions still remains unclear.

Particle deposition in human airways is a rather complicated process, and affected by many factors. These factors include particle size and shape, breathing rate, lung volume, and

\footnotetext{
* Corresponding author.

Tel.: +86-13487313916

E-mail address: oucuiyun@mail.sysu.edu.cn
}

health condition of the individual (Coates et al., 2001; Bui et al., 2020). Among them, particle size and breathing rate are the most important factors (Longest and Vinchurkar, 2007; Coates, 2008; Tsuda et al., 2013; Islam et al., 2017; Yu et al., 2020). Additionally, inhaled toxic airborne particles or pharmaceutical aerosols may transport and deposit in human respiratory systems due to different physical mechanisms: inertial impaction, gravitational sedimentation and Brownian diffusion (Hofmann, 2011; Hofemeier and Sznitman, 2015). However, the contributions of different mechanisms to particle deposition in airways vary with particle sizes and airflow rates. Therefore, it is important to identify the roles of particle size, airflow, and the mechanisms in the particle deposition in human airways.

Numerous studies have investigated the effects of mechanisms on particle deposition in human lung airways. Hofmann et al. (1995) demonstrated that gravitational settling may occur in peripheral airway bifurcations where both impaction and diffusion are not significant. Comer et al. (1999) found that inertial impaction is important for 
micron-sized particle deposition. Zhang et al. (2002) concluded that micron-sized particle deposition occurred mainly along the carinal ridges due to inertial impaction in a triplebifurcation airway model (G3-G6). Zhang et al. (2009) conducted another work by computing the micron-sized particle transport in a 16-generation model with repeated triple-bifurcation units in parallel and in series. They found that sedimentation in lower bronchial airways may change the impaction-caused deposition patterns. Farkas and Balásházy (2008) stressed that the deposition patterns were very sensitive to the particle size, and that particles larger than $1 \mu \mathrm{m}$ were mainly deposited due to impaction in the airway generations G0-G4. Kleinstreuer et al. (2007) found that both inertial impaction and gravitational sedimentation were significant mechanisms in the medium-sized bronchial bifurcations (G6-G9). Martonen et al. (2002) pointed out that particle deposition due to inertial impaction and sedimentation were non-linear functions of particle diameters in the human upper respiratory airway model. Therefore, the role of particle size and airflow in the deposition mechanism still remains uncertain.

The present study mainly focused on the particle size, airflow patterns and mechanisms of particle deposition in a three-dimensional (3-D) G3-G6 airway model. Using computational fluid dynamics (CFD), this work predicted the distribution of inhalable particles in different sizes delivered to the lung airways. Besides the particle diameter, the authors also considered various inhaling flow rates in an attempt to identify the mechanisms which affect the particle transport in the human lung airways.

\section{METHODS}

\section{Airway Geometry}

The human tracheobronchial tree is composed of a system of dichotomous tubular bifurcations (Horsfield and Thurlbeck, 1981). The bifurcation structure has a strong relation with both the airflow pattern and inhalable particle deposition in the human lung airways. Although the symmetric Weibel Type A (1963) airway configuration is not fully realistic, its 3-D bifurcating geometries can still be expected to generate accurate local deposition calculations. The first step of this study was to build a 3-D triplebifurcation geometry derived from the third to sixth generation of the Weibel (1963) symmetric model. For this purpose, the pre-processing tools Unigraphics (Version 5.0; Siemens PLM Software, Berlin, Germany) was used, taking into account the exact mathematical description of the morphologically realistic bifurcation model of Hegedüs $e t$ al. (2004). Then, a 3-D symmetric triple-bifurcation airway model is achieved and shown in Fig. 1, which also depicts the generation parameters.

\section{Governing Equations}

When the human body is in normal breathing state (from sleep to intense exercise), the respiratory rate of the oral cavity and/or nasal cavity is $3-60 \mathrm{~L} \mathrm{~min}^{-1}$. When airflow in this rate range enters the tracheobronchial airways concerned Therefore, in this work, the flow of the fluid is always laminar. airflow was assumed to remain laminar throughout the computed domain. Subsequently, for the three-dimensional

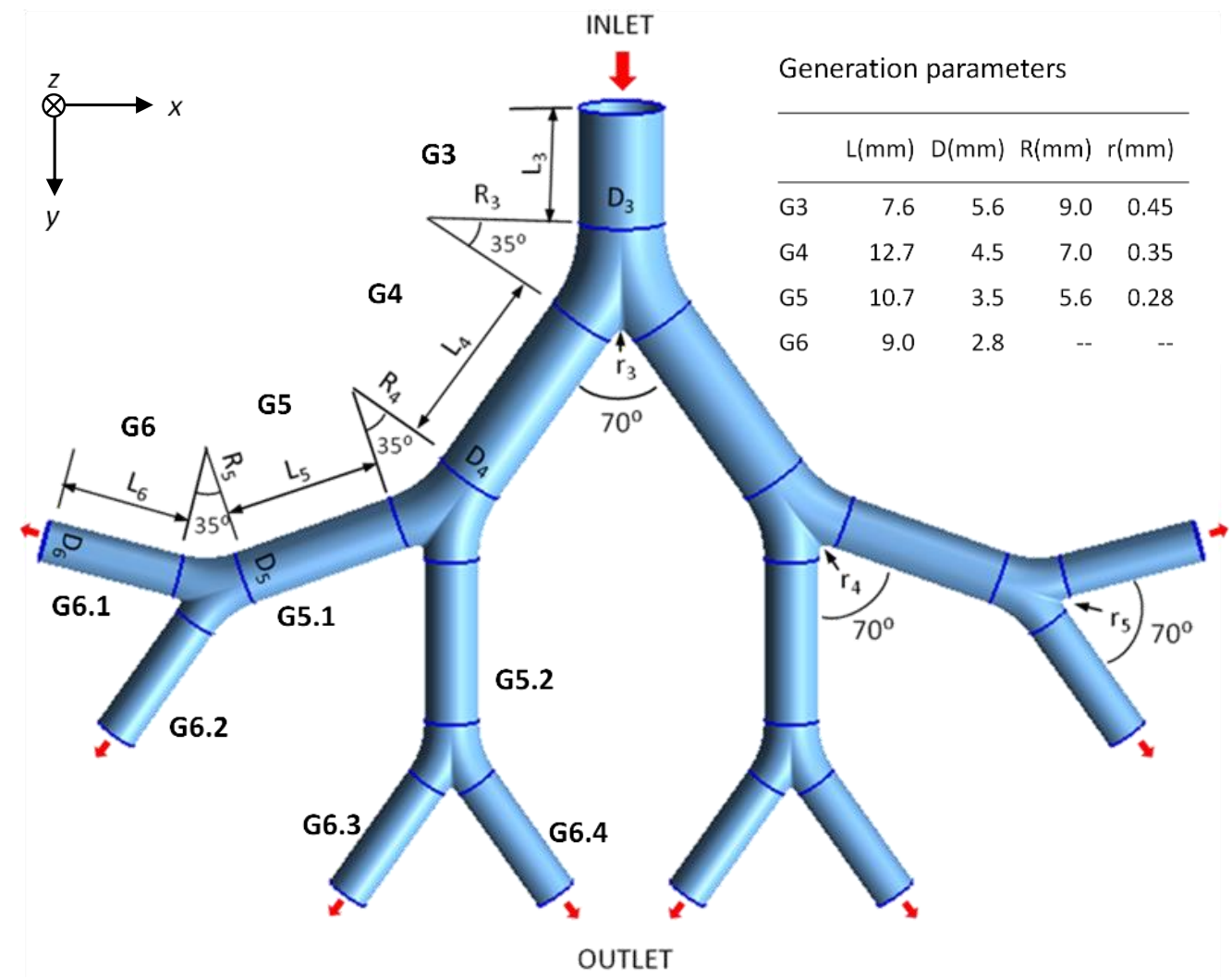

Fig. 1. Physical model of a triple-bifurcation unit of human lung airways (G3-G6). 
incompressible steady laminar flow in the rigid airways, the Navier-Stokes equations were given as follows:

$\nabla \cdot \mathbf{u}=0$

$\mathbf{u} \cdot \nabla \mathbf{u}=-\nabla \mathbf{p}+\frac{1}{\operatorname{Re}} \nabla^{2} \mathbf{u}$

where $\mathbf{u}$ and $\mathbf{p}$ are the velocity vector and pressure, respectively. All these variables are dimensionless and defined with the dimensional variables by $\mathbf{x}=x^{*} / \mathrm{D}_{3}, \mathbf{u}=$ $u^{*} / \mathrm{U}_{3}, \mathbf{p}=p^{*} /\left(\rho \mathrm{U}_{3}{ }^{2}\right)$, where $x^{*}=(x, y, z)$ is the Cartesian coordinate vector, $u^{*}=(u, v, w)$ represents the air velocity in three Cartesian coordinate directions, $D_{3}$ is the diameter of the inlet section (G3), $\mathrm{U}_{3}$ and $\rho$ are respectively the inlet velocity magnitude and the air density $\left(1.225 \mathrm{~kg} \mathrm{~m}^{-3}\right)$. The Reynolds number is defined as $\mathrm{Re}=\rho \mathrm{U}_{3} \mathrm{D}_{3} / \mu$, air dynamic viscosity $\mu=1.7894 \times 10^{-5} \mathrm{~kg}(\mathrm{~m} \mathrm{~s})^{-1}$. Computations are carried out for Reynolds numbers ranging from 100 to 2000 in the step of 100 , corresponding to the mean inlet velocity from 0.26 to $5.2 \mathrm{~m} \mathrm{~s}^{-1}$ (oral or nasal inhalation flow rate from 3 to $60 \mathrm{~L} \mathrm{~min}^{-1}$ from sleep to intense exercise).

Spherical particle trajectories are determined by postprocessing which is also called the one-way coupling method. Inertial impaction and gravitational sedimentation are the dominant mechanism for deposition in the upper bronchial airways and for micron-sized particles (Heyder, 2004). Brownian diffusion is the most important mechanism for deposition in the small airways and alveoli. Very fine particles $0.01 \mu \mathrm{m}$ or smaller are also trapped in the upper airway. The present work aims to investigate the $1-10 \mu \mathrm{m}$ particle deposition patterns in the upper airway model G3G6 where Brownian diffusion is negligible. Particles in the size ranging from 1 to $10 \mu \mathrm{m}$ are deposited mainly due to the inertial impaction and gravitational sedimentation. Thus, the drag and gravity are considered to be the dominant particle forces; then, the trajectory equation can be written as:

$$
\frac{d^{2} x_{p}^{*}}{d t^{2}}=f_{D}\left(u^{*}-u_{p}^{*}\right)+g
$$

where $x_{p}^{*}, u_{p}^{*}$ and $\mathrm{g}$ are the particle displacement, the particle velocity and the gravitational acceleration, respectively, and $f_{D}$ represents the drag force coefficient per unit particle mass given by:

$f_{D}=\frac{18 \mu}{\rho_{p} d_{p}^{2}} \frac{C_{D} \operatorname{Re}_{p}}{24}$

where $\rho_{\mathrm{p}}=2000 \mathrm{~kg} \mathrm{~m}^{-3}$ and $\mathrm{d}_{\mathrm{p}}$ are respectively the particle density and diameter, $\mathrm{C}_{\mathrm{D}}$ is the drag force coefficient (Haider and Levenspiel, 1989; Morsi and Alexander, 2006). The particle Reynolds number $\mathrm{Re}_{\mathrm{p}}$ is defined as:

$\operatorname{Re}_{p}=\frac{\rho d_{p}\left|u_{p}^{*}-u^{*}\right|}{\mu}$
$\mathrm{S}_{\mathrm{t}}$ is the Stokes number, and defined as:

$S_{t}=\frac{\rho_{p} d_{p}^{2} U_{3} C_{c}}{18 \mu D}$

where $\mathrm{C}_{\mathrm{c}}$ is the Cunningham correction factor.

\section{Boundary Conditions}

Uniform velocity profiles were applied at the inlet crosssection. Pressure-outlet boundary condition was adopted at the outlets. Non-slip boundary condition was employed along the airway walls.

In order to complete the particle transport formulations, the uniform-uniform inlet particle profile, according to Zhang and Kleinstreuer (2001), was adopted with the initial velocity of the corresponding airflow velocity. For all the walls, the trap condition was applied with the assumption that particles were trapped as soon as they touch the wall surfaces. Escape condition was applied to the inlet and outlets.

\section{Particle Deposition}

Deposition of particles in the human airways can be quantified in terms of the deposition efficiency (DE) in a specific region; they are defined as:

$D E=\frac{\text { Number of particles deposited in } \mathrm{Gi}}{\text { Number of particles entering } \mathrm{Gi}}$

In order to fulfill the aim of investigation on the impaction and sedimentation, three sub-parameters are introduced: $\mathrm{DE}_{\mathrm{i}}, \mathrm{DE}_{\mathrm{g}}$ and $\mathrm{DE}_{\mathrm{i}+\mathrm{g}}$. $\mathrm{DE}_{\mathrm{i}}$ is deposition efficiency due to the mechanism of inertial impaction; $\mathrm{DE}_{\mathrm{g}}$ refers to the deposition efficiency due to the gravitational sedimentation; $\mathrm{DE}_{\mathrm{i}+\mathrm{g}}$ means the deposition efficiency due to the combined mechanism of inertial impaction and gravitational sedimentation. The relations among $\mathrm{DE}_{\mathrm{i}}, \mathrm{DE}_{\mathrm{g}}$ and $\mathrm{DE}_{\mathrm{i}+\mathrm{g}}$ are given by the following equation derived by Yeh and Schum (1980):

$\mathrm{DE}_{\mathrm{i}+\mathrm{g}}=\mathrm{DE}_{\mathrm{i}}+\mathrm{DE}_{\mathrm{g}}-\mathrm{DE}_{\mathrm{i}} \cdot \mathrm{DE}_{\mathrm{g}}$

\section{Numerical Methods}

The governing equations and the associated boundary conditions were solved by using a finite volume method, employing the commercial CFD code FLUENT (Version 6.3; Ansys, Inc., Canonsburg, PA, USA). A structured mesh was generated by using GAMBIT (Version 2.4.6; Ansys, Inc.). Refined boundary layers were employed near walls and junctions where the velocity gradient may be large. For the grid independence test, we established three grid arrangements from coarse to fine, with cells of around $560,000,790,000$ and 1,600,000. As shown in Fig. 2, only the axial velocity along the middle cross-section of the branches in G5.1 and G5.2 was tested for the case of Re = 1000 in the present work. The results showed that the medium and fine grid arrangements can predict the axial velocity well with little discrepancy. Therefore, the medium 
grid arrangement for the symmetric geometry was selected to fulfill the subsequent validation and investigation.

The QUICK scheme was used to discretize the non-linear convection terms in the Navier-Stokes equation, which ensured an upwind character and high order accuracy. The segregated implicit approach was used to ensure a fast convergence and low memory requirement in order to obtain the steady solution. The standard scheme was used for the pressure gradients. The velocity field was obtained by solving momentum equations from an intermediate pressure field, and then the calculated velocity and continuity equations were modified by the SIMPLE algorithm. Relaxation factors of 0.7 and 0.3 were chosen for the velocity and pressure, respectively.

The discrete phase model (DPM) was used to calculate the particle trajectories after the steady airflow was achieved. To ensure independence from the number of particles released, different magnitudes were considered, and no significant change was found in deposition when the number was larger than 10,000 for all the cases considered.

\section{Model Validation}

A validation study has been carried out by comparing our results with the numerical data of Comer et al. (1999) and the experimental data of Kim and Fisher (1999). The results are all presented by deposition efficiency due to the combined mechanism of inertial impaction and gravitational sedimentation. As shown in Fig. 3, our simulation results are very close to both of the previous computational and experimental data, although there is little overestimation for $\mathrm{DE}$ of the first bifurcation and underestimation for DE of the second bifurcation. These small discrepancies can be attributed to the wider range of experimental Reynolds numbers (472 $\leq \mathrm{Re} \leq 2831$ ) beyond the laminar flow regime considered in the numerical method, experimental indeterminacy and small differences between the numerical geometry and experimental glass tube models.

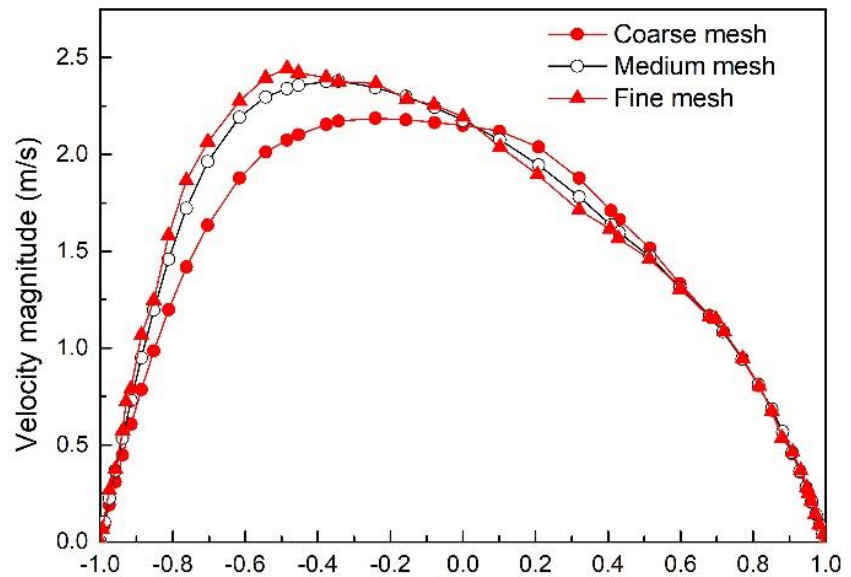

(a) G5.1

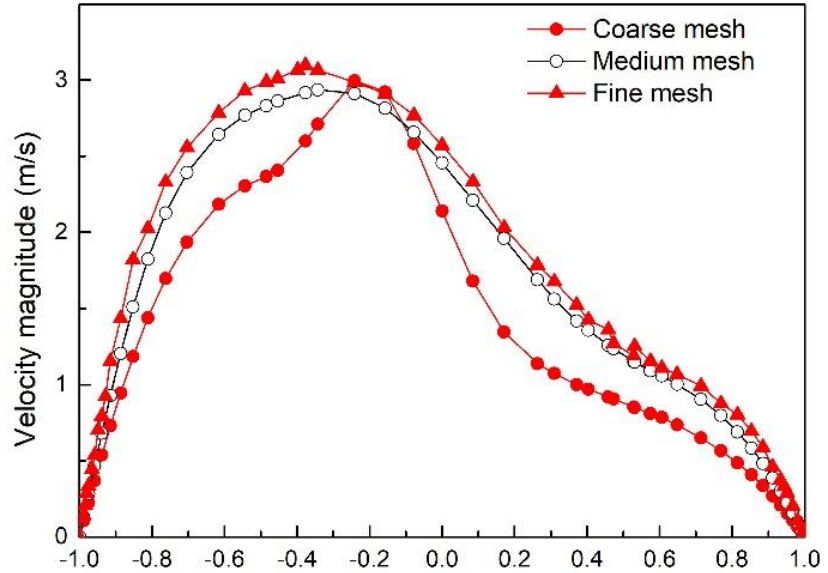

(b) G5.2

Fig. 2. Grid independence test: comparison of axial velocities along the cross-section of G5.1 and G5.2 among the coarse, medium and fine grid arrangements.

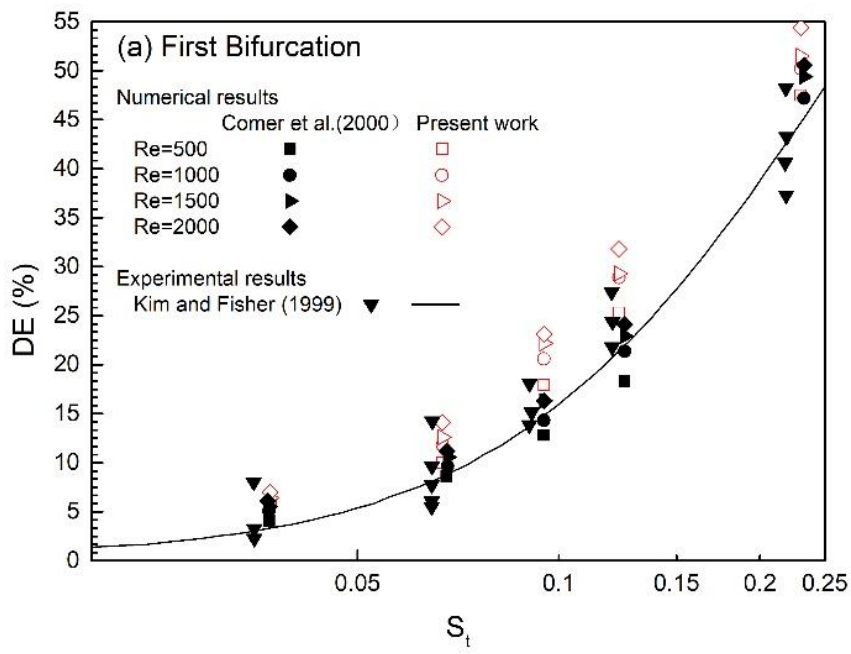

(a) First bifurcation

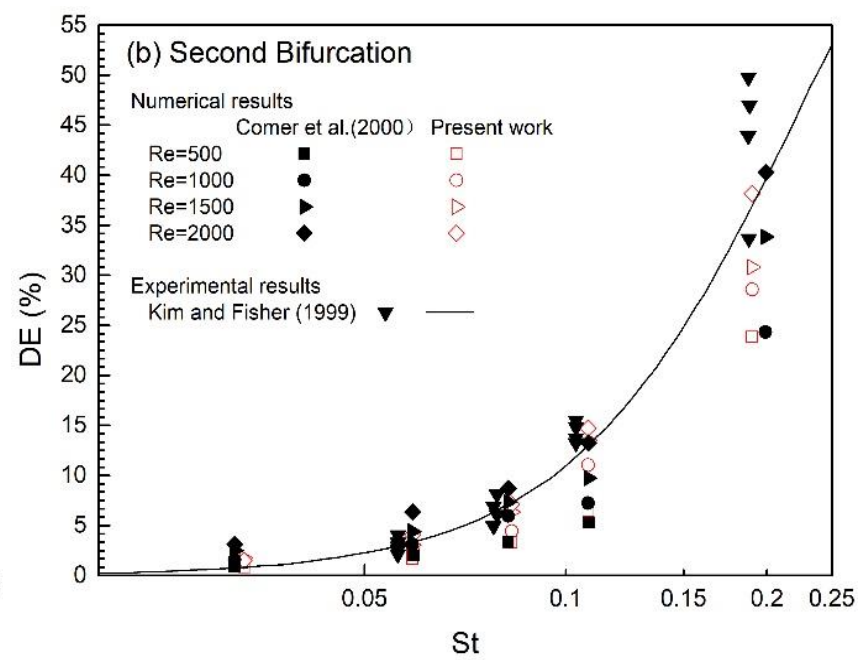

(b) Second bifurcation

Fig. 3. Comparisons between the present work and others for a double bifurcation: (a) first bifurcation and (b) second bifurcation. 


\section{RESULTS}

\section{Airflow Patterns}

Fig. 4 shows the overall view of axial and secondary airflow profiles and particle deposition patterns for the case $\operatorname{Re}=1000$ and $d p=10 \mu \mathrm{m}$. It shows that particle deposition is closely related to the airflow. Firstly, the incoming airflow splits at the first divider and a boundary layer appears at the inside wall of the first daughter tube (G4). Then, the daughter tube (G5.2) encounters a higher axial airflow rate than G5.1, which causes the daughter tube G6.3 to encounter the highest axial airflow rate among the tubes of G6. The secondary airflow is stronger in the tube where the axial airflow is higher and more non-uniform. For example, the secondary flow in $\mathrm{C}-\mathrm{C}^{\prime}$ cross-section of $\mathrm{G} 5.2$ is much stronger than that in $\mathrm{B}-\mathrm{B}^{\prime}$ of G5.1. On the other hand, the secondary airflow moves from the tube side where axial airflow is weaker to the side where axial airflow is stronger, i.e., $\mathrm{A} \rightarrow \mathrm{A}^{\prime}, \mathrm{B} \rightarrow \mathrm{B}^{\prime}, \mathrm{C} \rightarrow \mathrm{C}^{\prime}$, and so on. Particle deposition shown in Fig. 4 indicates that $10 \mu \mathrm{m}$ particles are mainly deposited in the tubes where the axial airflow rate is higher. Fig. 5 plots the axial and secondary flows in five crosssections of both G5.1 and G5.2 tubes for $\mathrm{Re}=1000$. It can be found that the secondary airflow is gradually weakened along the tube as the axial airflow gradually becomes homogeneous. The secondary flow and axial flow in G5.1 is much lower than that at the same level in G5.2. Along G5.1, the axial flow profile develops gradually from a rather skewed beginning to an almost parabolic ending, and the corresponding secondary flow weakens gradually. The number of vortices is reduced from two pairs to one pair, and the vortex cores migrate toward the center of the corresponding cross-section. Similar phenomena can be seen in the tube G5.2.

\section{Particle Deposition Patterns at the Upright Position}

Fig. 6 shows deposition efficiency of particles in the size range of $1-10 \mu \mathrm{m}$ due to different mechanisms under different Reynolds numbers. In general, particle deposition efficiency increases with particle size. Specifically, $D_{i}$ increases rapidly (Fig. 6(a)) while $\mathrm{DE}_{\mathrm{g}}$ increases log-linearly with particle size (Fig. 6(b)). On the other hand, the effect of Re is different. $\mathrm{DE}_{\mathrm{i}}$ increases rapidly with $\mathrm{Re}$ while $\mathrm{DE}_{\mathrm{g}}$ decreases; $\mathrm{DE}_{\mathrm{i}+\mathrm{g}}$ appears similar with $\mathrm{DE}_{\mathrm{g}}$ when $\mathrm{Re} \leq 200$, and similar with $\mathrm{DE}_{\mathrm{i}}$ when $\mathrm{Re} \geq 500$ and $\mathrm{dp} \geq 3 \mu \mathrm{m}$ (Fig. 6(c)). Hence in the subsequent sections, we will investigate the deposition patterns for $1,3,5$ and $10 \mu \mathrm{m}$ particles under the Reynolds number of 100, 200, 500, 1000, 1500 and 2000.

Fig. 7 displays the variation of deposition efficiency in terms of non-dimensional parameters. Fig. 7(a) shows the variation of $D E_{i}$ with $S_{t}$. DE $E_{i}$ varies according to the same pattern during the entire range of Re. It shows a consistent curve, i.e., an $S$-shaped growth curve, and can be fitted into a logistic regression curve as $\mathrm{DE}_{\mathrm{i}}(\%)=100\left(1-1 /\left(63.05 \mathrm{~S}_{\mathrm{t}}^{2.36}\right.\right.$ $+1)$ ). Fig. 7(b) illustrates the variation of $\mathrm{DE}_{\mathrm{g}}$ in terms of $\mathrm{S}_{\mathrm{t}} / \mathrm{Re}^{2}$. The values of the gravitational deposition efficiency can be fitted to a logistic regression curve as $\operatorname{DE}_{\mathrm{g}}(\%)=$ $100\left(1-1 /\left(2 \times 10^{6}\left(\mathrm{~S}_{\mathrm{t}} / \mathrm{Re}^{2}\right)^{1.127}+1\right)\right)$

Fig. 8 shows the deposition efficiency in the whole model of G3-G6 due to different mechanisms for 1, 3, 5 and $10 \mu \mathrm{m}$ particles. For particles in all of the four considered sizes, $\mathrm{DE}_{\mathrm{i}}$ increases with $\mathrm{Re}$ while $\mathrm{DE}_{\mathrm{g}}$ decreases. The dominant mechanisms of deposition for particles in different sizes are

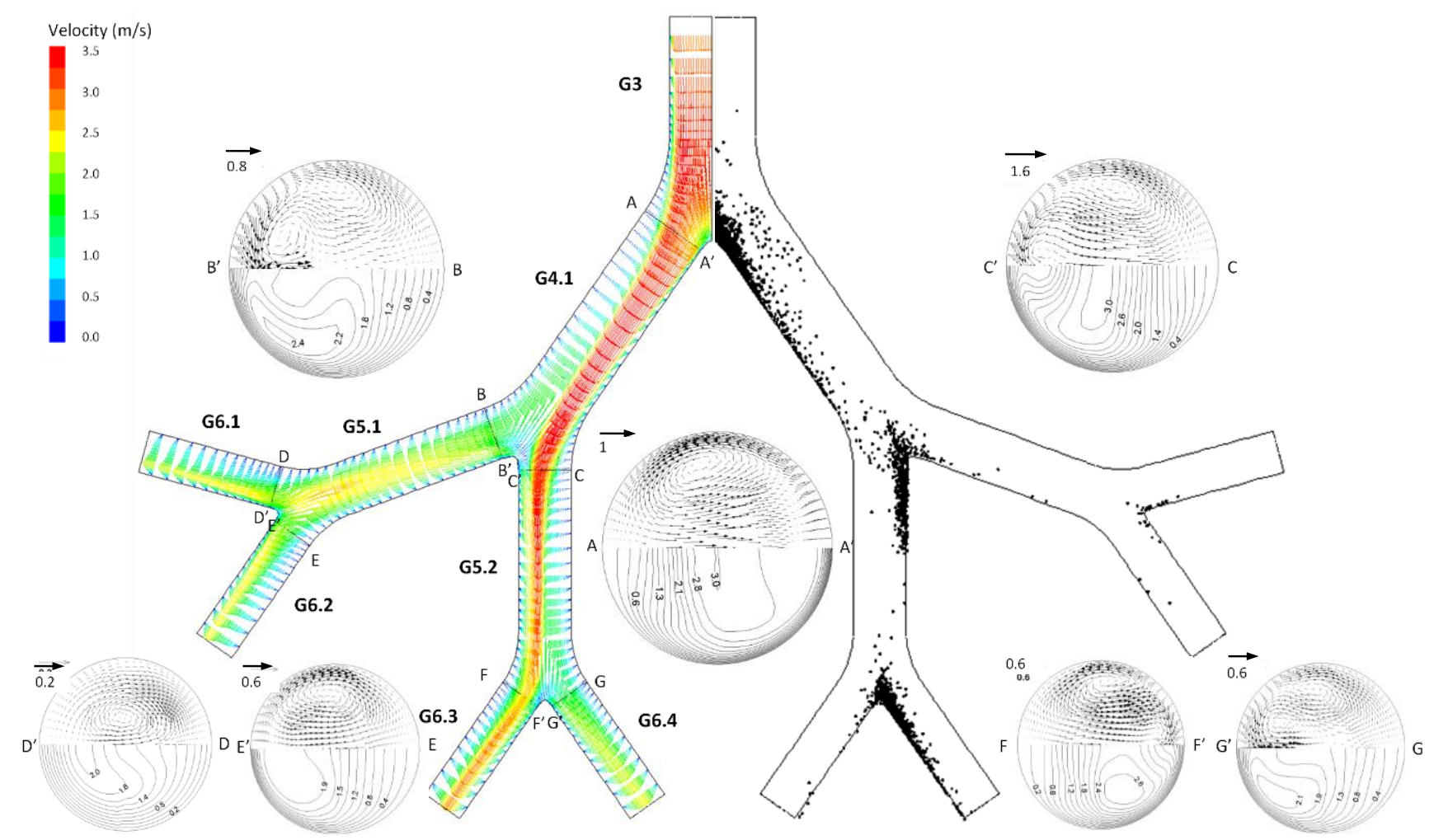

Fig. 4. Axial and secondary velocity distributions and particle deposition pattern for $\operatorname{Re}=1000$ and $\mathrm{dp}=10 \mu \mathrm{m}$. 


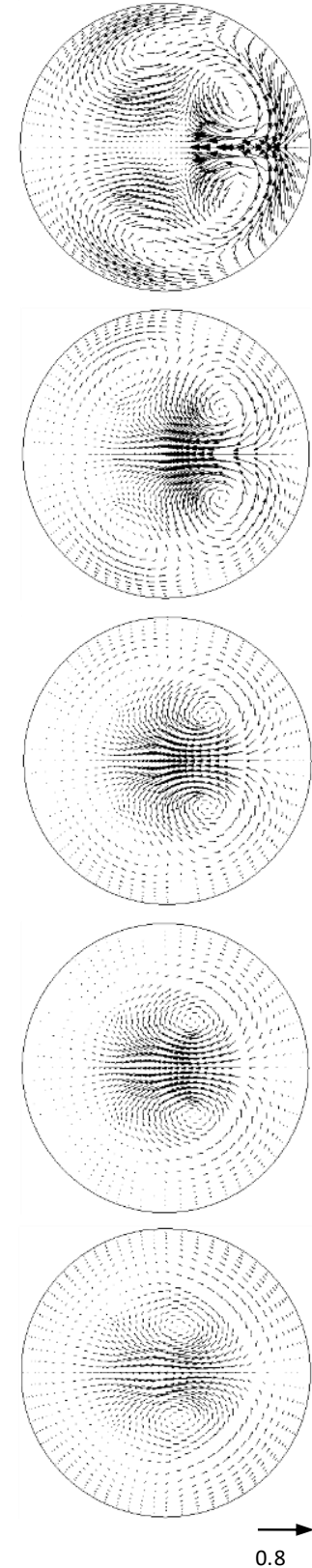

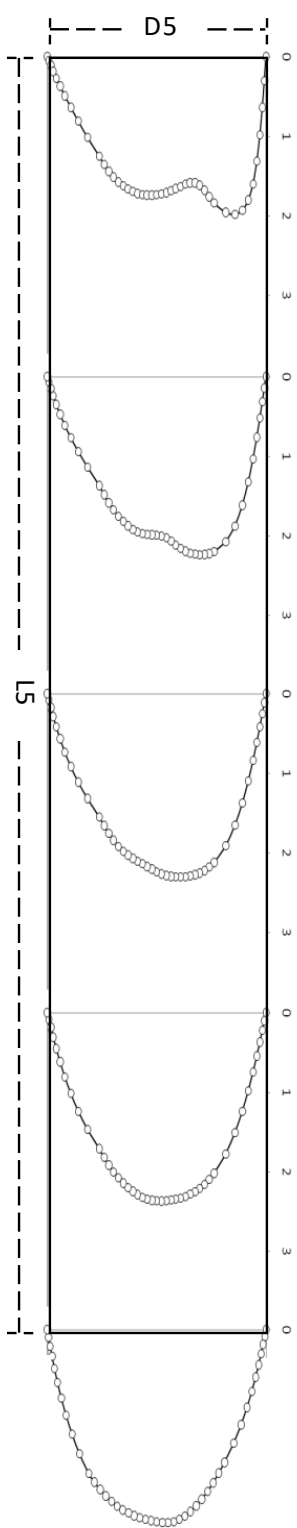

Axial velocity

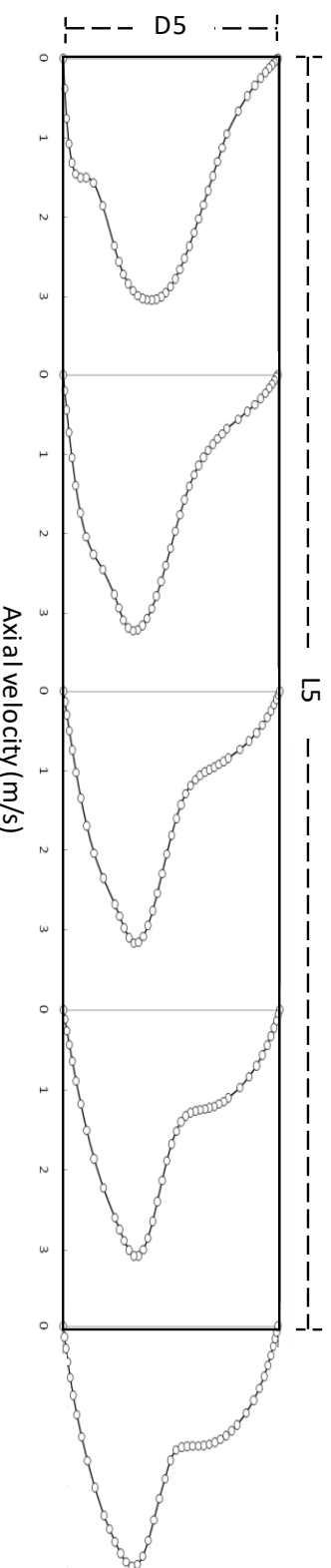

Axial velocity
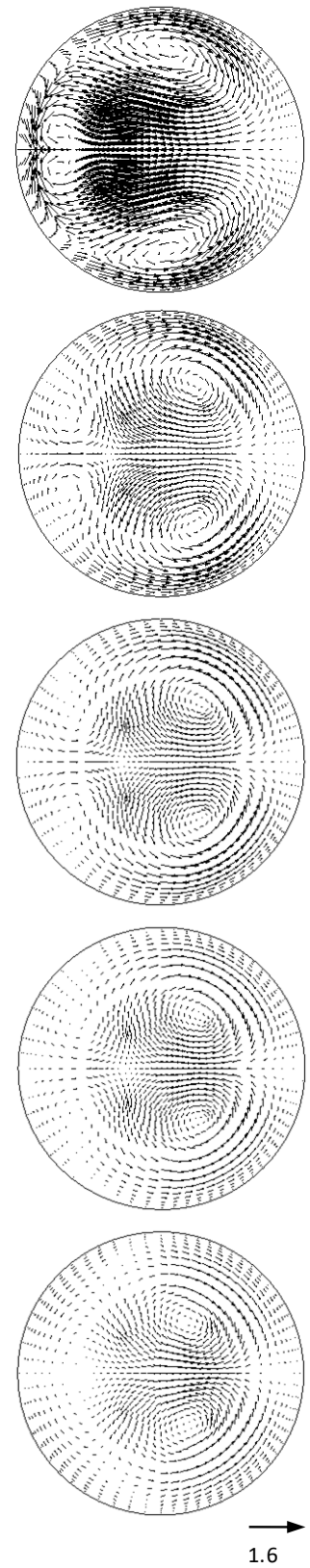

Secondary velocity

(a) G5.1 (B $\left.\rightarrow B^{\prime}\right)$

(b) $\mathrm{G} 5.2\left(\mathrm{C}^{\prime} \rightarrow \mathrm{C}\right)$

Fig. 5. Axial and secondary velocity distributions for the generation G5 for $\operatorname{Re}=1000$, (a) G5.1, and (b) G5.2.

different. For small particles $(\mathrm{dp}=1 \mu \mathrm{m})$, gravitational sedimentation dominates, and the deposition efficiency is low. For medium-sized particles, balance between gravity and inertia can be found: There is a $U$-shaped $\mathrm{DE}_{\mathrm{i}+\mathrm{g}}$ curve for $3 \mu \mathrm{m}$ particles. Firstly, the particle deposition efficiency decreases rapidly with the increasing Reynolds numbers when the gravitational sedimentation dominates. Then, it keeps nearly constant due to the balance between inertial impaction and gravitational sedimentation. Finally, the $\mathrm{DE}_{\mathrm{i}+\mathrm{g}}$ curve rapidly increases with Re. For large particles (dp =
$5 \mu \mathrm{m}$ and $10 \mu \mathrm{m}$ ), the curve of $\mathrm{DE}_{\mathrm{i}+\mathrm{g}}$ is like a right hand of $U$ shape as inertial impaction dominates even at low Reynolds numbers.

Fig. 9 shows the 2-D views of particle deposition patterns due to different mechanisms under four Reynolds numbers. As particles deposit symmetrically at the first carina due to the symmetric airway, half of the airway model for each case separated by the $x=0$ plane is displayed. As the deposition efficiency of 1 and $3 \mu \mathrm{m}$ particles is too low $(<1 \%$ and $<7 \%$, respectively; Figs. 8(a) and 8(b)), the 2-D views of their 


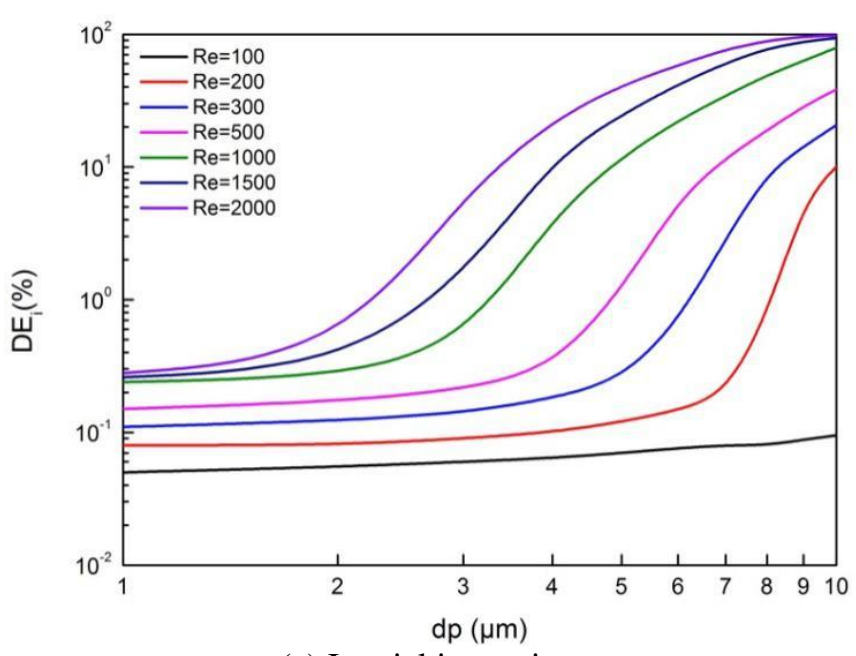

(a) Inertial impaction

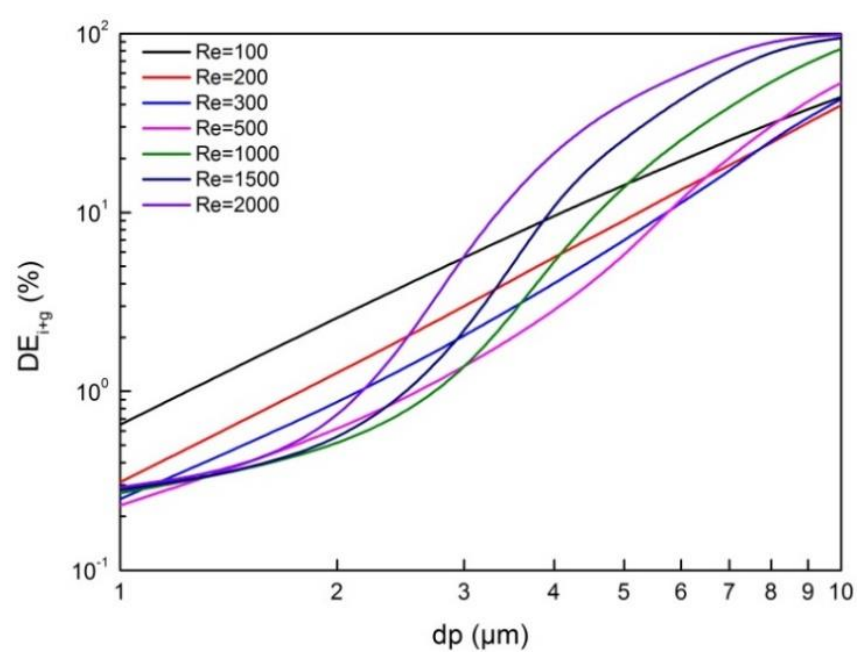

(c) Combined mechanism

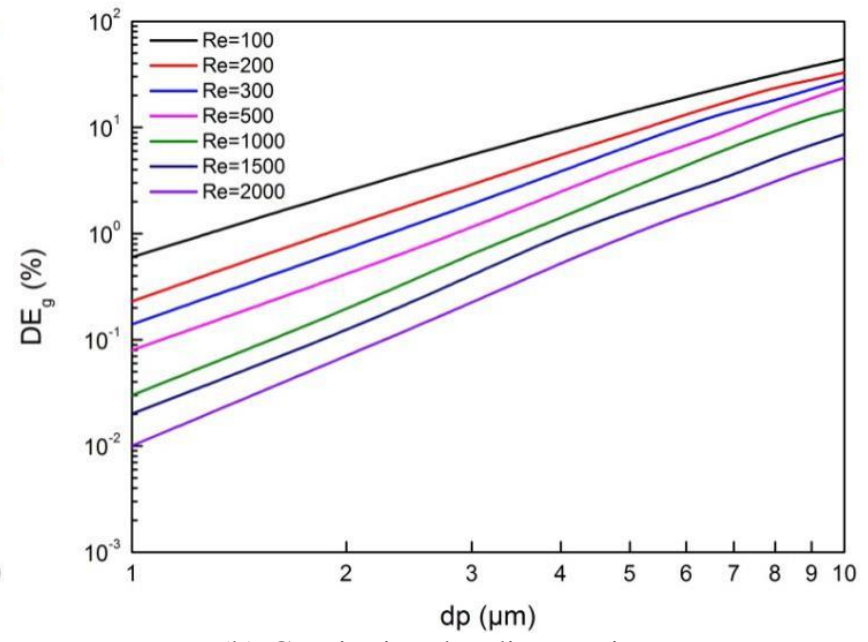

(b) Gravitational sedimentation

Fig. 6. Particle deposition efficiency at different Reynolds numbers vs. particle diameters due to different mechanisms: (a) inertial impaction, (b) gravitational sedimentation, and (c) combined mechanism.

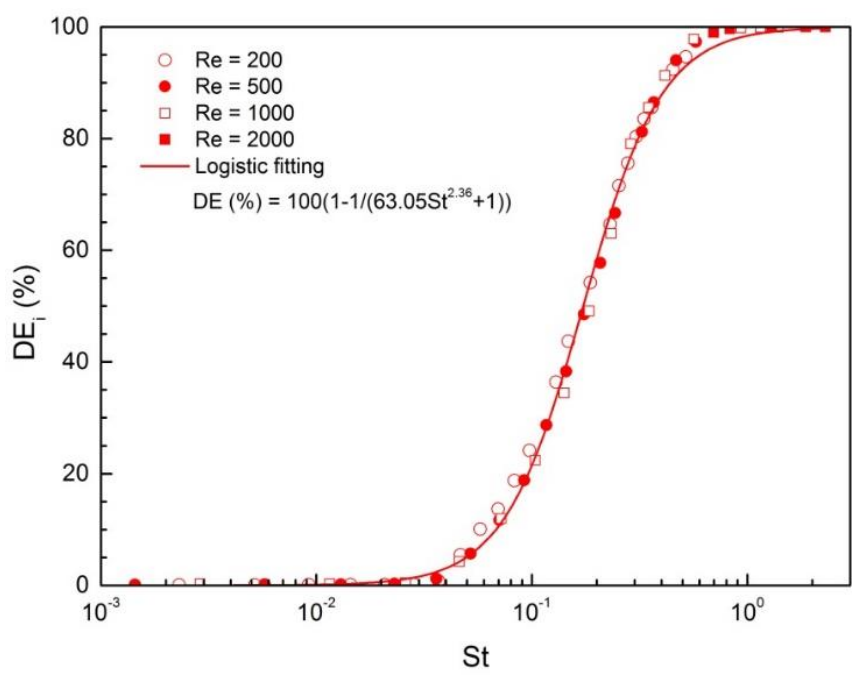

(a) Inertial impaction

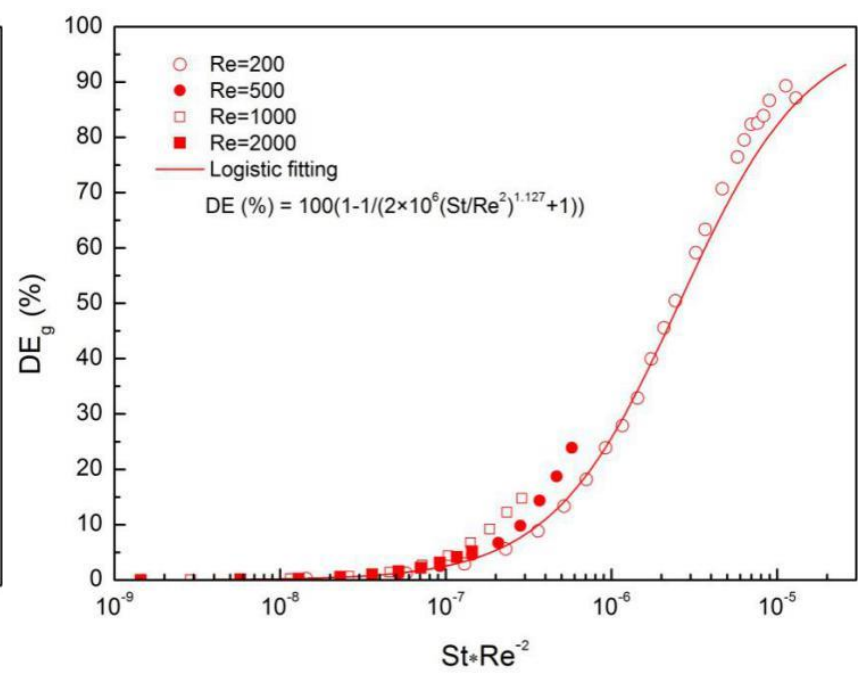

(b) Gravitational sedimentation

Fig. 7. Particle deposition efficiency at different Reynolds numbers vs. non-dimensional parameters due to different deposition mechanisms: (a) inertial impaction and (b) gravitational sedimentation. 


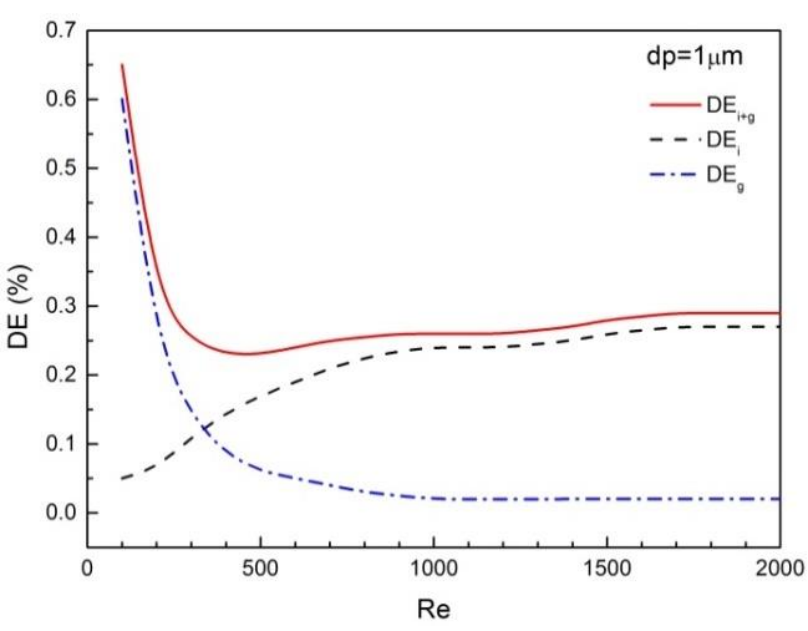

(a) $\mathrm{dp}=1 \mu \mathrm{m}$

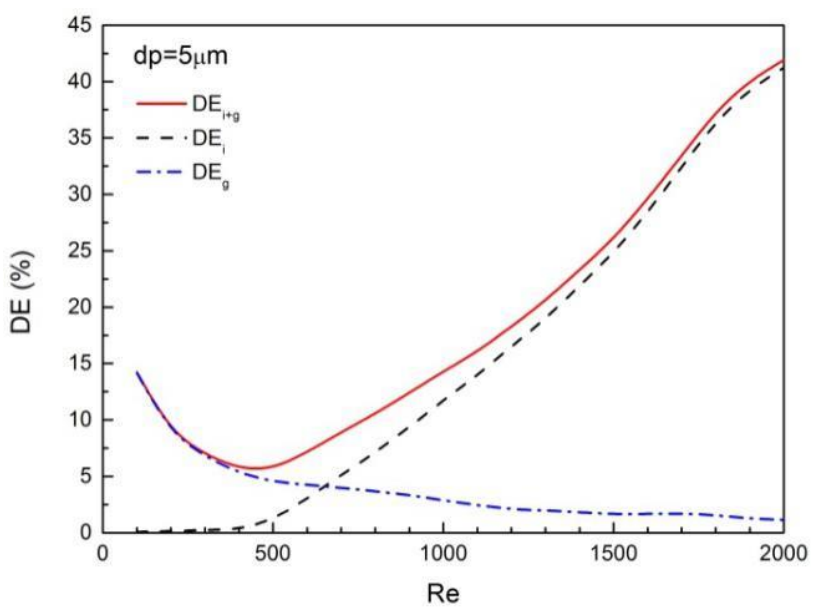

(c) $\mathrm{dp}=5 \mu \mathrm{m}$

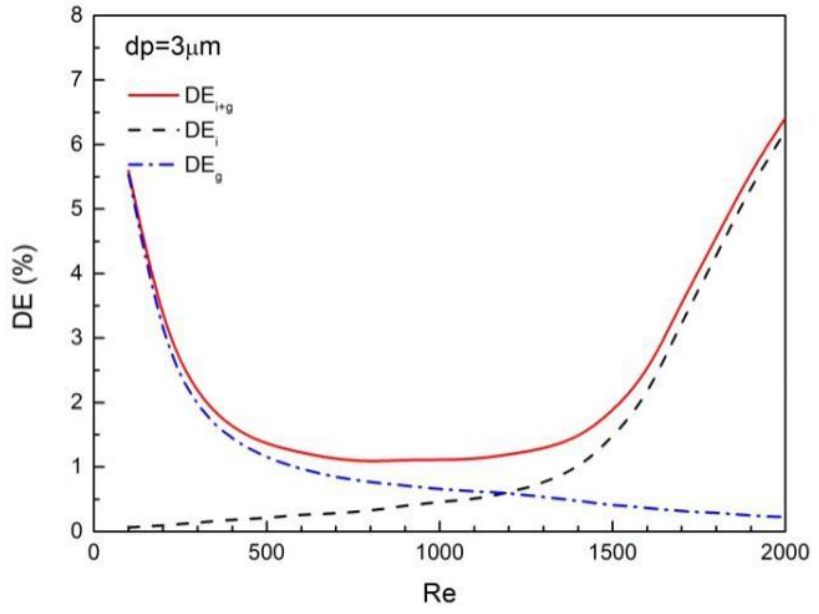

(b) $\mathrm{dp}=3 \mu \mathrm{m}$

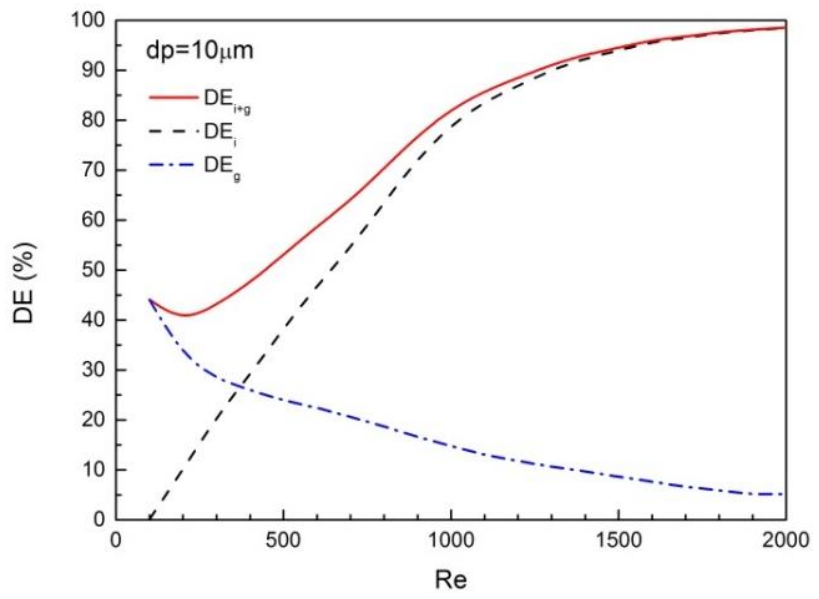

(d) $\mathrm{dp}=10 \mu \mathrm{m}$

Fig. 8. Deposition efficiency (DE) under different mechanisms for particles of different diameters: $(\mathrm{a}) \mathrm{dp}=1 \mu \mathrm{m}$, $(\mathrm{b}) \mathrm{dp}=$ $3 \mu \mathrm{m}$, (c) $\mathrm{dp}=5 \mu \mathrm{m}$, and (d) $\mathrm{dp}=10 \mu \mathrm{m}$.

deposition patterns are not shown here. Only cases of $\mathrm{dp}=$ $5 \mu \mathrm{m}$ and $\mathrm{dp}=10 \mu \mathrm{m}$ are illustrated. As shown in the figure, nearly no deposition can be found in the left panel of (a), which indicates that inertial impaction is not effective yet at $\operatorname{Re}=100$, while for the combined mechanism (the right panel (a)), particles distribute uniformly along the inside wall of tubes without hot spots for both particle diameters due to gravitational sedimentation. When Re rises, the effects of gravitational sedimentation decease, while the inertial impaction becomes more and more effective, and significant hot spots can be found at carinas due to inertial impaction. At $\operatorname{Re}=2000$, particles are deposited mainly by inertial mechanism that results in non-uniform deposition along the inside wall of tubes with hot spot sites around the carinae.

\section{Particle Deposition Patterns at Different Body Positions}

Fig. 10 shows the particle deposition efficiency at different human body orientations for particles in different sizes (dp $=3 \mu \mathrm{m}$ and $10 \mu \mathrm{m}$ ). Four human body orientations are considered for the comparison, upright posture, handstand position, lateral position, and dorsal position, which means four different directions relative to gravity. We assume that G3 and the plane of bifurcations are parallel to the gravity when the human body is in the upright posture. As shown in Fig. 10(a), changing the body orientation can lead to different deposition patterns for both small $(\mathrm{dp}=3 \mu \mathrm{m})$ and large (dp $=10 \mu \mathrm{m})$ particles. For $3 \mu \mathrm{m}$ particles, the handstand position holds the lowest $\mathrm{DE}$ for $\mathrm{Re}<1800$. The upright and lateral positions have similar DE for all the considered Re. The dorsal position has the highest DE in the Re range of 5001700. For $10 \mu \mathrm{m}$ particles, the difference among DE under different postures decreases with Re. The lowest DE lies in the case of the handstand position for $\mathrm{Re}<700$ and the dorsal position for $\mathrm{Re}>1000$. The upright posture has the highest DE for nearly all Re.

\section{DISCUSSION}

This study was to predict the effect of particle size and airflow rate and the contribution of different mechanisms to particle deposition in a triple-bifurcation airway model. It is found that the particles in different size can be deposited due to different mechanism. 

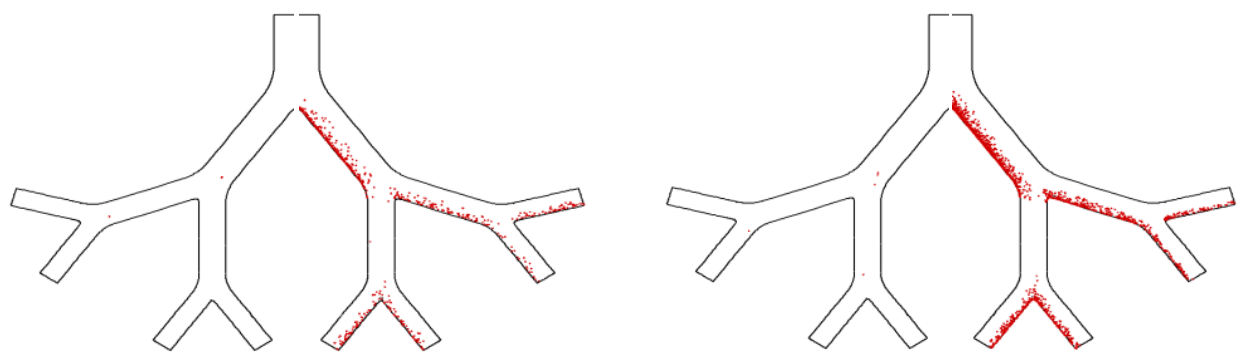

(a) $\mathrm{Re}=100$
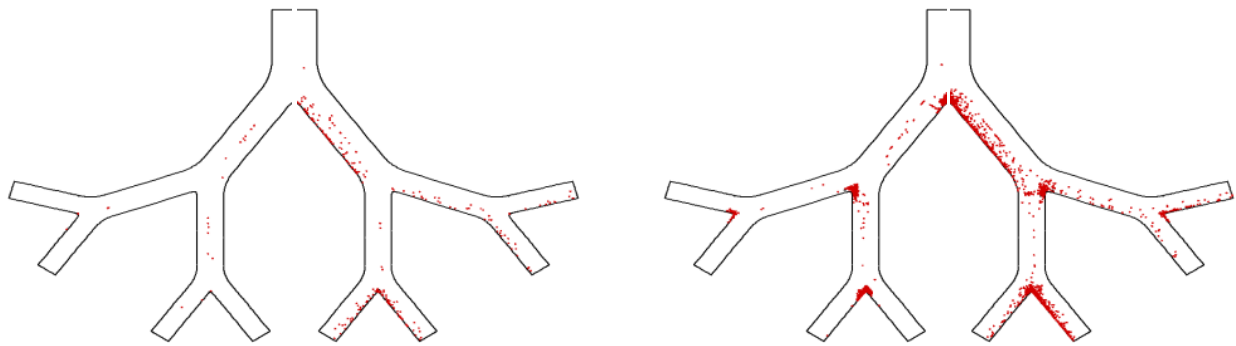

(b) $\mathrm{Re}=500$
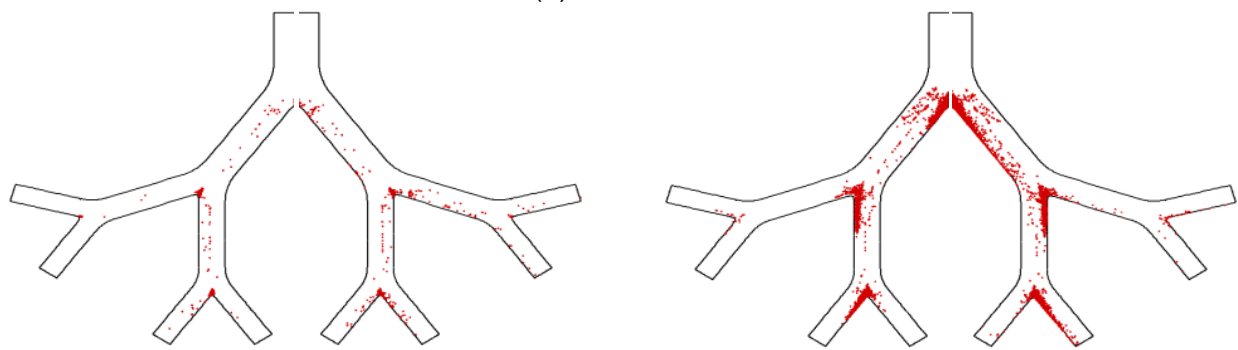

(c) $\mathrm{Re}=1000$
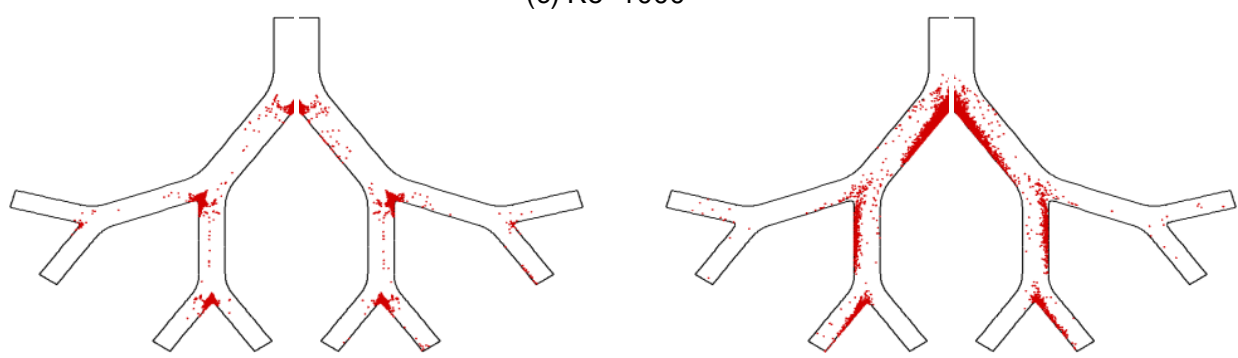

(d) $\mathrm{Re}=2000$

Fig. 9. Particle deposition patterns for $\mathrm{dp}=5 \mu \mathrm{m}$ (left column) and $\mathrm{dp}=10 \mu \mathrm{m}$ (right column) under different mechanisms (left half: inertial; right half: inertial + gravitational) for different Reynolds numbers: (a) $\operatorname{Re}=100,(b) \operatorname{Re}=500,(c) \operatorname{Re}=$ 1000 , and (d) $\operatorname{Re}=2000$.

The present work used the three-dimensional in-plane triple-bifurcation airway model (G3-G6). Longest and Xi (2007) investigated the inertial impaction on deposition of fine and ultrafine particles in two double-bifurcation airway models: G3-G5 and G7-G9. They found that inertia was more significant than previously considered for the deposition of particles in the range. However, Comer et al. (1999) showed that double bifurcations may not provide a realistic view of deposition in respiratory airways. Therefore, more generations are necessary (Yang et al., 2006). Kleinstreuer and Zhang (2008) suggested that triple-bifurcation airway model provided a feasible and accurate approach to predict deposition in individual bifurcations.

The airflow structure was found to significantly affect the particle deposition patterns. Firstly, the airway geometry, especially the structure of the dividers between generations, could influence the airflow fields. Then, $10 \mu \mathrm{m}$ particles deposited more at sites where airflow rates were higher. It is also observed more particles depositing at the inside of bifurcations due to the secondary flow. It was because the vortex drove the low-speed flow and particles from the outside toward the inside of bifurcations. Chen et al. (2018a) also stressed that $\mathrm{PM}_{2.5}$ deposition was mainly due to inertial impaction in the asthmatic airways as a result of the secondary flow. Li et al. $(2007 \mathrm{~b}, \mathrm{c})$ also suggested that the spatial configuration of airways significantly influenced velocity structure and thus resulted in different particle distributions. Rahimi-Gorji et al. (2016) found that the maximum velocity change in the larynx may cause more particles to deposit in this site. 
(a)

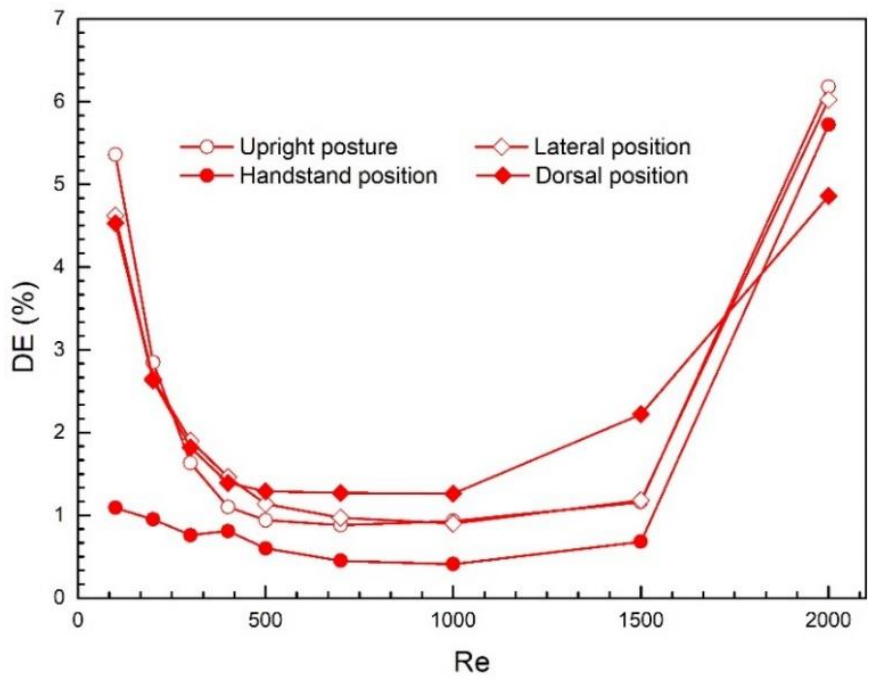

(b)

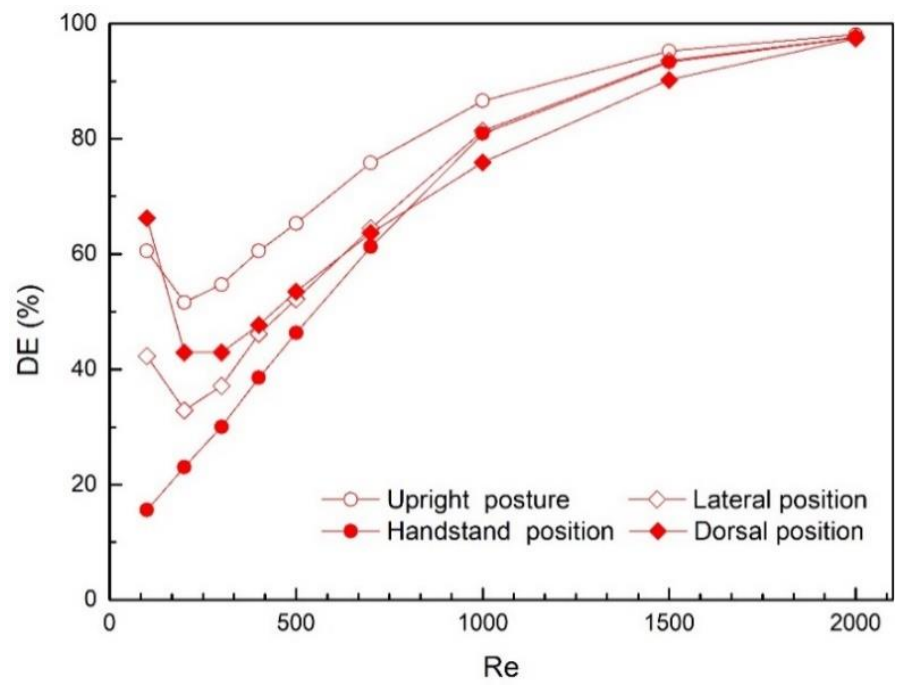

Fig. 10. Particle deposition efficiency at different human body orientations for particles in different sizes: (a) $d p=3 \mu \mathrm{m}$ and (b) $\mathrm{dp}=10 \mu \mathrm{m}$.

The strength of this study lies in the fact that the authors have separated the role of different mechanism of deposition in the airway model. The deposition mechanism is one of the factors that determined the particle deposition patterns in the human airways (Hofmann, 2011). Particles deposit in the lung mainly due to the following three mechanisms: inertial impaction, gravitational sedimentation and Brownian diffusion. Previous studies have already demonstrated that particles can deposit due to different mechanisms in the simple bifurcation airway models (Lee et al., 1996; Kleinstreuer et al., 2007; Longest and Xi, 2007). However, they did not show the precise fraction which mechanism burdens in the deposition of particles in different sizes. Kleinstreuer et al. (2007) indicated that sedimentation deposition was significantly amplified during slow inhalation (i.e., $\mathrm{dp}>5 \mu \mathrm{m}$ at $\mathrm{Q}_{\text {in }}=3.75 \mathrm{~L} \mathrm{~min}^{-1}$ for the generations G6G9). This work investigated the variation of $\mathrm{DE}_{\mathrm{i}}, \mathrm{DE}_{\mathrm{g}}$ and $\mathrm{DE}_{\mathrm{i}+\mathrm{g}}$ in terms of Re and particle size. It was found that $\mathrm{DE}_{\mathrm{g}}$ decreased with $\mathrm{Re}$ while $\mathrm{DE}_{\mathrm{i}}$ increased with Re. This work defined the lowest Reynolds number when inertial impaction dominates over gravitational sedimentation as the critical value of Re. It was found that the critical value would shift from high $\left(\operatorname{Re}_{\text {critical }}=1500\right.$ for $\left.\mathrm{dp}=3 \mu \mathrm{m}\right)$ to low $\left(\operatorname{Re}_{\text {critical }}=300\right.$ for $\left.\mathrm{dp}=10 \mu \mathrm{m}\right)$ with the increasing particle size. It meant that inertial impaction for larger particles would dominate at lower Reynolds number. Consequently, the bigger the particles are, the more they will deposit at the proximal airways.

The other strength of the study is that the authors established the relationships between non-dimensional parameters and $\mathrm{DE}_{\mathrm{i}}$ as well as $\mathrm{DE}_{\mathrm{g}}$. Firstly, it was found that $D_{i}$ varied with $S_{t}$ in a consistent regression curve for the entire range of inlet $\mathrm{Re}$ and obtained a statistical regression equation in terms of $S_{t}$. Then, $\mathrm{DE}_{\mathrm{g}}$ was found to vary with the ratio of $\mathrm{S}_{\mathrm{t}}$ to $\mathrm{Re}^{2}$ in a consistent way. Cheng et al. (2015) conducted an experiment by using replicas of the human mouth and throat. They found that $\mathrm{DE}_{\mathrm{i}+\mathrm{g}}$ had a good correlation with $S_{t}$ only, because the inertial impaction dominated over gravity when flow rates ranged from 15 to $60 \mathrm{~L} \mathrm{~min}^{-1}$ (Cheng et al., 2015). Kleinstreuer et al. (2007) 
suggested that $\mathrm{DE}_{\mathrm{i}+\mathrm{g}}$ in the airway model of the generations G6-G9 could be correlated only with $S_{t}$ under normal breathing conditions (i.e., $\mathrm{Q} \geq 15 \mathrm{~L} \mathrm{~min}^{-1}$ ). They also found that the sedimentation became increasingly dominant over inertial impaction under slow breathing conditions (i.e., $\mathrm{Q} \leq$ $7.5 \mathrm{~L} \mathrm{~min}^{-1}$ ) (Kleinstreuer et al., 2007). It means that the regression of DE should not take into account $S_{t}$ only when flow rate is slow. Chen et al. (2018b) suggested that deposition in the airway model of the generations G11-G14 were determined by $S_{t}$ and the sedimentation parameter for particles in the size range of $1-7 \mu \mathrm{m}$, which agrees well with our present results.

Another merit is that the present work investigated the influence of changes of postures on the particle deposition in G3-G6 airway model. Four different orientations were investigated in this work: upright posture, handstand position, lateral position, and dorsal position. Hofmann et al. (1995) stressed that the gravity angle could significantly determine the local and regional deposition patterns in the G15-G16 human airway model. Harrington et al. (2006) also indicated that orientations of the gravity to the airway model may affect the $1-5 \mu \mathrm{m}$ particle deposition patterns in the G18-G22 airway model. Tao et al. (2018) conducted a 2-D CFD simulation to investigate the effect of gravity direction on the 1-3 $\mu \mathrm{m}$ particle deposition in pulmonary acinus during breath holding. They found that gravitational direction had no effect on the total deposition fraction, but could change the local deposition (Tao et al., 2018). This work found that the change of human body orientation could influence the deposition patterns, except that the upright and lateral positions held similar DE for $3 \mu \mathrm{m}$ particles under all the inlet Re.

There are several limitations in the present study. Firstly, the symmetric in-plane triple-bifurcation airway model was not realistic, while realistic airway model was more sufficient for the simulation than the symmetric model (Sul et al., 2014; Xi et al., 2016). Secondly, a realistic, fluctuating inlet velocity profile, i.e., the parabolic profile, should be considered in future work (Mutuku and Chen, 2018). It is because the inlet airflow patterns can affect particle deposition (Park and Wexler, 2008; Lai et al., 2013; Rahimi-Gorji et al., 2015; Phuong et al., 2016). Thirdly, the particle characteristics should be considered in the future work, as they (e.g., hygroscopicity and shape) may greatly affect the deposition amount as well as the sites. Moreover, the particle release patterns (random parabolic, random uniform, random random, and determined parabolic) also affect the particle deposition patterns (Zhang and Kleinstreuer, 2001; Li et al., 2007a), which certainly warrants further study.

\section{CONCLUSIONS}

This study employed a human airway model (G3-G6) and CFD to comprehensively analyze the deposition of inhalable micron-sized $(1 \mu \mathrm{m} \leq \mathrm{dp} \leq 10 \mu \mathrm{m})$ particles in the tracheobronchial tree. We found that the particle size and inhalation rate determined the site, amount, and mechanism (inertial impaction or gravitational sedimentation) of deposition, with most of the accumulation occurring on the inner walls of the tubes that were subjected to a higher axial flow rate and stronger inward secondary velocity. Furthermore, neither inertial impaction nor gravitational sedimentation allowed the size fraction less than $3 \mu \mathrm{m}$ in diameter to settle in the G3-G6 airways. We also discovered that inertial impaction replaced gravitational sedimentation as the dominant deposition mechanism once the airflow rate (represented by the Reynolds number [Re]) reached a critical value, which decreased as the particle size increased. Therefore, larger particles $(\mathrm{dp}>3 \mu \mathrm{m})$, which were deposited via inertial impaction rather than gravitational sedimentation even when Re was low, mainly settled in the proximal bifurcations. The orientation of the human body was revealed as another potential factor in the pattern of deposition, although the upright and lateral positions exhibited similar deposition efficiencies for $3 \mu \mathrm{m}$ particles regardless of Re. Overall, the deposition due to inertial impaction and that due to gravitational sedimentation primarily depended on the Stokes number $\left(S_{t}\right)$ and the ratio of $S_{t}$ to $R^{2}$, respectively. These findings identify the critical Reynolds number at which the particle deposition mechanism for a specific size shifts from gravitation to inertia.

\section{ACKNOWLEDGEMENTS}

The research was supported by the China Postdoctoral Science Foundation (No. 2019M653147), the National Natural Science Foundation of China (No. 42005069, No. 51576214, No. 41875015, and No. 51811530017), Fundamental Research Funds for the Central Universities of China (Sun Yat-Sen University, 18lgpy08), the Natural Science Foundation of Guangdong Province (No. 2018A030310307), and the National Natural Science Foundation-Outstanding Youth Foundation (Grant No. 41622502).

\section{REFERENCES}

Bui, V.K.H., Moon, J.Y., Chae, M., Park, D. and Lee, Y.C. (2020). Prediction of aerosol deposition in the human respiratory tract via computational models: A review with recent updates. Atmosphere 11: 137. https://doi.org/10.33 90/atmos 11020137

Chen, W.H., Lee, K.H., Mutuku, J.K. and Hwang, C.J. (2018a). Flow dynamics and $\mathrm{PM}_{2.5}$ deposition in healthy and asthmatic airways at different inhalation statuses. Aerosol Air Qual. Res. 18: 866-883. https://doi.org/10.4 209/aaqr.2018.02.0058

Chen, X., Feng, Y., Zhong, W., Sun, B. and Tao, F. (2018b). Numerical investigation of particle deposition in a triple bifurcation airway due to gravitational sedimentation and inertial impaction. Powder Technol. 323: 284-293. https://doi.org/10.1016/j.powtec.2017.09.050

Cheng, Y.S., Zhou, Y. and Su, W.C. (2015). Deposition of particles in human mouth-throat replicas and a USP induction port. J. Aerosol Med. Pulm. Drug Deliv. 28: 147-155. https://doi.org/10.1089/jamp.2013.1105

Coates, A.L. (2008). Guiding aerosol deposition in the lung. N. Engl. J. Med. 358: 304-305. https://doi.org/10.1056/N EJMcibr0707489 
Coates, A.L., Allen, P.D., MacNeish, C.F., Ho, S.L. and Lands, L.C. (2001). Effect of size and disease on estimated deposition of drugs administered using jet nebulization in children with cystic fibrosis. Chest 119: 1123-1130. https://doi.org/10.1378/chest.119.4.1123

Comer, J.K., Kleinstreuer, C., Hyun, S. and Kim, C.S. (1999). Aerosol transport and deposition in sequentially bifurcating airways. J. Biomech. Eng. 122: 152-158. https://doi.org/10.1115/1.429636

Deng, Q., Ou, C., Chen, J. and Xiang, Y. (2018). Particle deposition in tracheobronchial airways of an infant, child and adult. Sci. Total Environ. 612: 339-346. https://doi.org/10.1016/j.scitotenv.2017.08.240

Deng, Q., Ou, C., Shen, Y.M., Xiang, Y., Miao, Y. and Li, Y. (2019). Health effects of physical activity as predicted by particle deposition in the human respiratory tract. Sci. Total Environ. 657: 819-826. https://doi.org/10.1016/j.s citotenv.2018.12.067

Farkas, Á. and Balásházy, I. (2008). Quantification of particle deposition in asymmetrical tracheobronchial model geometry. Comput. Biol. Med. 38: 508-518. https://doi.org/10.1016/j.compbiomed.2008.01.014

Finlay, W.H. and Martin, A.R. (2008). Recent advances in predictive understanding of respiratory tract deposition. $J$. Aerosol Med. Pulm. Drug Deliv. 21: 189-206. https://doi.org/10.1089/jamp.2007.0645

Haider, A. and Levenspiel, O. (1989). Drag coefficient and terminal velocity of spherical and nonspherical particles. Powder Technol. 58: 63-70. https://doi.org/10.1016/0032 -5910(89)80008-7

Harrington, L., Kim Prisk, G. and Darquenne, C. (2006). Importance of the bifurcation zone and branch orientation in simulated aerosol deposition in the alveolar zone of the human lung. J. Aerosol Sci. 37: 37-62. https://doi.org/10. 1016/j.jaerosci.2005.03.005

Hegedüs, C.J., Balásházy, I. and Farkas, Á. (2004). Detailed mathematical description of the geometry of airway bifurcations. Respir. Physiol. Neurobiol. 141: 99-114. https://doi.org/10.1016/j.resp.2004.03.004

Heyder, J. (2004). Deposition of inhaled particles in the human respiratory tract and consequences for regional targeting in respiratory drug delivery. Proc. Am. Thorac Soc. 1: 315-320. https://doi.org/10.1513/pats.200409-046TA

Hofemeier, P. and Sznitman, J. (2015). Revisiting pulmonary acinar particle transport: Convection, sedimentation, diffusion, and their Interplay. J. Appl. Physiol. 118: 13751385. https://doi.org/10.1152/japplphysiol.01117.2014

Hofmann, W., Balásházy, I. and Koblinger, L. (1995). The effect of gravity on particle deposition patterns in bronchial airway bifurcations. J. Aerosol Sci. 26: 11611168. https://doi.org/10.1016/0021-8502(95)00044-D

Hofmann, W. (2011). Modelling inhaled particle deposition in the human lung-A review. J. Aerosol Sci. 42: 693724. https://doi.org/10.1016/j.jaerosci.2011.05.007

Horsfield, K. and Thurlbeck, A. (1981). Relation between diameter and flow in branches of the bronchial tree. Bull. Math. Biol. 43: 681-691. https://doi.org/10.1007/BF0245 8417

Islam, M.S., Saha, S.C., Sauret, E., Gemci, T. and Gu, Y.T.
(2017). Pulmonary aerosol transport and deposition analysis in upper 17 generations of the human respiratory tract. J. Aerosol Sci. 108: 29-43. https://doi.org/10.1016/ j.jaerosci.2017.03.004

Kim, C.S. and Fisher, D.M. (1999). Deposition characteristics of aerosol particles in sequentially bifurcating airway models. Aerosol Sci. Technol. 31: 198-220. https://doi.org/10.1080/027868299304255

Kleinstreuer, C., Zhang, Z. and Kim, C.S. (2007). Combined inertial and gravitational deposition of microparticles in small model airways of a human respiratory system. $J$. Aerosol Sci. 38: 1047-1061. https://doi.org/10.1016/j.jaer osci.2007.08.010

Kleinstreuer, C. and Zhang, Z. (2008). An adjustable triplebifurcation unit model for air-particle flow simulations in human tracheobronchial airways. J. Biomech. Eng. 131: 021007-021007-021010. https://doi.org/10.1115/1.3005339

Lai, T.C., Morsi, Y.S., Das, S. and Owida, A. (2013). Numerical analysis of particle deposition in asymmetrical human upper airways under different inhalation cycles. $J$. Mech. Med. Biol. 13: 1350068. https://doi.org/10.1142/S 0219519413500681

Lee, J.W., Goo, J.H. and Chung, M.K. (1996). Characteristics of inertial deposition in a double bifurcation. J. Aerosol Sci. 27: 119-138. https://doi.org/10.1016/0021-8502(95) 00538-2

Li, Z., Kleinstreuer, C. and Zhang, Z. (2007a). Particle deposition in the human tracheobronchial airways due to transient inspiratory flow patterns. J. Aerosol Sci. 38: 625-644. https://doi.org/10.1016/j.jaerosci.2007.03.010

Li, Z., Kleinstreuer, C. and Zhang, Z. (2007b). Simulation of airflow fields and microparticle deposition in realistic human lung airway models. Part I: Airflow patterns. Eur. J. Mech. B. Fluids. 26: 632-649. https://doi.org/10.1016/ j.euromechflu.2007.02.003

Li, Z., Kleinstreuer, C. and Zhang, Z. (2007c). Simulation of airflow fields and microparticle deposition in realistic human lung airway models. Part II: particle transport and deposition. Eur. J. Mech. B. Fluids. 26: 650-668. https://doi.org/10.1016/j.euromechflu.2007.02.004

Löndahl, J., Wollmer, P., Gudmundsson, A., Nicklasson, H., Swietlicki, E. and Rissler, J. (2017). Measurement of respiratory tract deposition of inhaled particles $(0.015-5$ $\mu \mathrm{m})$, lung function and breathing pattern for a group of 67 adults and children. Eur. Respir. J. 50: PA2638. https://doi.org/10.1183/1393003.congress-2017.PA2638

Longest, P.W. and Vinchurkar, S. (2007). Effects of mesh style and grid convergence on particle deposition in bifurcating airway models with comparisons to experimental data. Med. Eng. Phys. 29: 350-366. https://doi.org/10.1016/j.m edengphy.2006.05.012

Longest, P.W. and Xi, J. (2007). Computational investigation of particle inertia effects on submicron aerosol deposition in the respiratory tract. J. Aerosol Sci. 38: 111130. https://doi.org/10.1016/j.jaerosci.2006.09.007

Martonen, T.B., Zhang, Z., Yue, G. and Musante, C.J. (2002). 3-D Particle transport within the human upper respiratory tract. J. Aerosol Sci. 33: 1095-1110. https://doi.org/10.1016/S0021-8502(02)00060-5 
Morsi, S.A. and Alexander, A.J. (2006). An investigation of particle trajectories in two-phase flow systems. J. Fluid Mech. 55: 193-208. https://doi.org/10.1017/S002211207 2001806

Mutuku, J.K. and Chen, W.H. (2018). Flow characterization in healthy airways and airways with chronic obstructive pulmonary disease (COPD) during different inhalation conditions. Aerosol Air Qual. Res. 18: 2680-2694. https://doi.org/10.4209/aaqr.2018.06.0232

Park, S.S. and Wexler, A.S. (2008). Size-dependent deposition of particles in the human lung at steady-state breathing. J. Aerosol Sci. 39: 266-276. https://doi.org/10. 1016/j.jaerosci.2007.11.006

Patton, J.S. and Byron, P.R. (2007). Inhaling medicines: Delivering drugs to the body through the lungs. Nat. Rev. Drug Discovery 6: 67-74. https://doi.org/10.1038/nrd2153

Phuong, N.L., Yamashita, M., Yoo, S.J. and Ito, K. (2016). Prediction of convective heat transfer coefficient of human upper and lower airway surfaces in steady and unsteady breathing conditions. Build. Environ. 100: 172 185. https://doi.org/10.1016/j.buildenv.2016.02.020

Rahimi-Gorji, M., Pourmehran, O., Gorji-Bandpy, M. and Gorji, T.B. (2015). CFD simulation of airflow behavior and particle transport and deposition in different breathing conditions through the realistic model of human airways. J. Mol. Liq. 209: 121-133. https://doi.org/10.10 16/j.molliq.2015.05.031

Rahimi-Gorji, M., Gorji, T.B. and Gorji-Bandpy, M. (2016). Details of regional particle deposition and airflow structures in a realistic model of human tracheobronchial airways: Two-phase flow simulation. Comput. Biol. Med. 74: 1-17. https://doi.org/10.1016/j.compbiomed.2016.04. 017

Sul, B., Wallqvist, A., Morris, M.J., Reifman, J. and Rakesh, V. (2014). A computational study of the respiratory airflow characteristics in normal and obstructed human airways. Comput. Biol. Med. 52: 130-143. https://doi.org/ 10.1016/j.compbiomed.2014.06.008

Tao, W., Hongyan, Z. and Haihang, C. (2018). Numerical simulation of the effect of gravitational direction on particle deposition in pulmonary acinus under the condition of breath holding. Nanotechnol. Precis. Eng. 1: 66-72. https://doi.org/10.13494/j.npe.20170003

Tsuda, A., Henry, F.S. and Butler, J.P. (2013). Particle transport and deposition: Basic physics of particle kinetics. Compr. Physiol. 3: 1437-1471. https://doi.org/1 0.1002/cphy.c100085

Xi, J., Yuan, J.E., Yang, M., Si, X., Zhou, Y. and Cheng, Y.S. (2016). Parametric study on mouth-throat geometrical factors on deposition of orally inhaled aerosols. J. Aerosol Sci. 99: 94-106. https://doi.org/10.1016/j.jaerosci.2016.0 1.014

Yang, X.L., Liu, Y., So, R.M.C. and Yang, J.M. (2006). The effect of inlet velocity profile on the bifurcation COPD airway flow. Comput. Biol. Med. 36: 181-194. https://doi.org/10.1016/j.compbiomed.2004.11.002

Yeh, H.C. and Schum, G.M. (1980). Models of human lung airways and their application to inhaled particle deposition. Bull. Math. Biol. 42: 461-480. https://doi.org/10.1016/S0092-8240(80)80060-7

Yu, H., Yen, H.L. and Li, Y. (2020). Deposition of bronchiole-originated droplets in the lower airways during exhalation. J. Aerosol Sci. 142: 105524. https://doi.org/10.1016/j.jaerosci.2020.105524

Zhang, Z. and Kleinstreuer, C. (2001). Effect of particle inlet distributions on deposition in a triple bifurcation lung airway model. J. Aerosol Med. 14: 13-29. https://doi.org/10.1089/08942680152007864

Zhang, Z., Kleinstreuer, C. and Kim, C.S. (2002). Cyclic micron-size particle inhalation and deposition in a triple bifurcation lung airway model. J. Aerosol Sci. 33: 257281. https://doi.org/10.1016/S0021-8502(01)00170-7

Zhang, Z., Kleinstreuer, C. and Kim, C.S. (2009). Comparison of analytical and CFD models with regard to micron particle deposition in a human 16-generation tracheobronchial airway model. J. Aerosol Sci. 40: 16-28. https://doi.org/10.1016/j.jaerosci.2008.08.003

Received for review, February 19, 2020

Revised, August 24, 2020

Accepted, August 27, 2020 